\title{
A Comprehensive Analysis of Genetic Diversity and Relationships of 13 Sweet Sorghum Varieties as Energy Sources"
}

\author{
Qiuwen Zhan, Ling Zhou, Ningning Bi, Haocheng Wu, Jieqin Li, Jie Lu, Jingbiao Lu, Ping Lin \\ Anhui Science and Technology University, Fengyang, China \\ Email: qwzhan@163.com
}

Received September 11, 2012; revised October 15, 2012; accepted October 23, 2012

\begin{abstract}
To investigate the genetic diversity and relationships among the sweet sorghum varieties as energy sources currently bred in China, 13 sweet sorghum varieties were selected for comprehensive analysis through observations of 31 biological traits and examinations of RAPD and SSR molecular markers. The numerical analysis showed that the differences in biological traits existed among 13 varieties, and the genetic distance (DIST) ranged from 0.787 to 2.221, and the two varieties from Inner Mongolia and Xinjiang were distinctly separated from all other varieties. A total of 22 polymorphism primers were obtained from the screening using RAPD marker analysis. The polymorphism rate was $58.33 \%$, and the genetic similarity (GS) coefficients among the studied cultivars ranged from 0.694 to 0.896. Cluster analysis results indicated that the three varieties from Inner Mongolia, Xinjiang and Heilongjiang exhibited significant genetic differences from the other varieties. SSR marker analysis using 31 selected pairs of polymorphic primers showed that the polymorphism rate of amplified fragments was $78.64 \%$, and GS coefficients among the tested cultivars were 0.534 to 0.971 . Cluster analysis showed that variety No. 12 from Xinjiang and variety No. 7 from Inner Mongolia clustered into one group, and variety No. 6 from Heilongjiang was in a single group. The other ten varieties were grouped into another separate cluster. The results based on combined data displayed a similar trend with results from the three individual data analyses, but could more comprehensively and objectively reflect the fundamental genetic differences among these varieties. In summary, certain genetic differences exist among the varieties tested from different regions or different breeding institutions. However, varieties from the same region, especially those from the same breeding institution, exhibited small genetic variations and high genetic similarities. At present, more attention should be paid to discovery and innovation in the breeding of sweet sorghum varieties.
\end{abstract}

Keywords: Sweet Sorghum as Energy Source; RAPD; SSR; Genetic Diversity; Cluster Analysis

\section{Introduction}

Sweet sorghum [Sorghum bicolor (L.) Moench] belongs to the genus Sorghum in the family Gramineae. It is a variant of common grain sorghum, with characteristics of resistance to drought, flood, barren soil and soil salinity and alkalinity. The stem of sweet sorghum is rich in sugar; it can be used as fodder or a sugar source and can be converted into alcohol to use as an energy source. Two to three times more carbohydrates are synthesized by sweet sorghum than by corn or beets. In ethanol production, the degradation and saccharification of starch is reduced when using sweet sorghum instead of corn or cassava, which significantly reduces the production cost. Due to an increasing worldwide shortage of mineral and

*This work was supported by the National Natural Science Foundation of China (No: 31071470) and Transformation Fund for Agricultural Science and Technology Achievements of China (No: 2008GB2C300125). energy resources, sweet sorghum shows potential as an energy crop due to its high biological yield. As a renewable energy crop, sweet sorghum has received broad attention from domestic breeding institutions in China. In the last two years, the number of varieties that have participated in the national sweet sorghum variety regional trials has dramatically increased compared to previous years, with 13 - 14 varieties in 2008-2010. The increase in bred varieties is well-received progress, and it is worth investigating the genetic diversity of these varieties because this diversity is indicative of the innovation in sweet sorghum germplasm resources and the future direction of variety breeding in China.

Numerical classification based on morphology has become an effective way to test the genetic relationships of species [1,2]. Random amplification of polymorphic DNA (RAPD) marker analysis based on molecular biology requires only a simple assay, easy sample collection, small 
amounts of DNA and use of universal primers [3]; therefore, it has been widely used in the analysis of genetic diversity and relationships, phylogenetics and characterization of the purity of hybrid seeds [3]. Simple sequence repeat (SSR) marker analysis also has several advantages, such as simple and fast operation, high sensitivity, good stability and abundant allele polymorphisms. Therefore, it has been widely employed in crop germplasm identification, genetic mapping, gene mapping and genetic diversity analysis [4-6]. However, no reports have been published on studies of the genetic diversity of sweet sorghum varieties bred in China. This study aimed to analyze genetic diversity among the 13 sweet sorghum varieties enrolled in the national regional trial via numerical analysis, RAPD and SSR molecular markers to understand the genetic relationships between these currently popular sweet sorghum varieties in China and to provide a theoretical basis for the utilization and innovation of sweet sorghum germplasm resources.

\section{Materials and Methods}

\subsection{Experimental Materials}

The names and sources of the materials are listed in Table 1.

\subsection{Field Experimental Design and Trait Observation}

Field experiments were carried out in the Planting Technology Park of Anhui Science and Technology University. The 13 varieties were planted in a randomized block design in triplicate. The sowing date was April 30, 2008, and the harvest date was September 18, 2008. During the entire growth period, 31 biological traits of sweet sorghum were surveyed including 23 quantitative traits and 8 qualitative traits. Analyses were performed following the methods in reference [1].

\subsection{Molecular Biology Methods}

DNA extraction and RAPD PCR reaction conditions were as previously described [7]. SSR PCR reactions were performed as described [6] with modifications.

\subsection{Data Analysis}

\subsubsection{Numerical Analysis}

From the 23 quantitative traits, 5 important agronomic traits affecting energy usage were selected for variance analysis between the varieties. All of the 31 traits were used for cluster analysis, of which 23 quantitative traits were directly involved in the computation of clustering and 8 qualitative traits were used in computations after specific coding. All data were processed using NTSYS-pc Version 2.10e [8]. The standardized matrix was processed using the Interval Data of Similarity subroutine to obtain the DIST matrix. The Unweighted Pair Group Method with Arithmetic Mean (UPGMA) clustering method of the Clustering subroutine SAHN was used to construct the dendrogram in operational taxonomic units.

\subsubsection{RAPD and SSR Electrophoresis Data Analysis} A molecular marker data matrix was established by recording 1 for the presence of a specific band and 0 for the absence of the band. NTSYS-pc was used, in which the measure of similarity coefficient was SM. The UPGMA method was used for cluster analysis and phylogenetic tree construction [8].

Table 1. Name and origin of the varieties used in this study.

\begin{tabular}{rll}
\hline No & \multicolumn{1}{c}{ Variety Name } & \\
\hline 1 & Liaotian No. 4 & The Sorghum Institute, Liaoning Academy of Agricultural Sciences \\
2 & Liaotian No. 5 & The Sorghum Institute, Liaoning Academy of Agricultural Sciences \\
3 & Jinsiza No. 1 & Jinzhou Institute of Agricultural Sciences, Liaoning Province \\
4 & Pac8381 & Beijing Doneed Seeds (Chifeng) Co, Ltd \\
5 & Jintian No. 1 & The Sorghum Institute, Shanxi Academy of Agricultural Sciences \\
6 & Longtian 062 & The Crop Breeding Institute, Heilongjiang Academy of Agricultural Sciences \\
7 & Chitian No. 1 & Chifeng Institute of Agricultural Sciences, Inner Mongolia \\
8 & Liaotian No. 7 & The Sorghum Institute, Liaoning Academy of Agricultural Sciences \\
9 & Liaotian No. 6 & The Sorghum Institute, Liaoning Academy of Agricultural Sciences \\
10 & BT802 & Shenyang Laoben Seeds Co, Ltd, Liaoning Province \\
11 & Shenshi 202 & The Crop Institute, Shenyang Academy of Agricultural Sciences, Liaoning Province \\
12 & Xingaoliang No. 3 & The Economic Crop Institute, Xinjiang Academy of Agricultural Sciences \\
13 & Liaosiza No. 1 & The Sorghum Institute, Liaoning Academy of Agricultural Sciences \\
\hline
\end{tabular}




\subsubsection{Combined Data Analysis}

The 31 surveyed biological traits and the $0-1$ data of the RAPD and SSR electrophoretic bands were used to construct a combined data matrix. NTSYS-pc software was used for analysis [8]. After standardization using subroutine Standardization (standard deviation, STD), the DIST distance was obtained. Then, the UPGMA method was employed for cluster analysis of the 13 varieties based on the combined data.

\section{Results and Analysis}

\subsection{Numerical Analysis and Cluster Analysis Based on Biological Traits}

Among the 13 varieties, variance analysis of 5 major agronomic traits showed no significant differences in stem diameter at the mature stage $\left(F=0.51<F_{0.05}\right)$ or in the number of tillings $\left(F=0.98<F_{0.05}\right.$ ), but revealed a highly significant difference in plant height at the mature stage $\left(F=15.76>F_{0.01}\right)$ with variety No. 7 displaying a highly significant difference from the other 12 varieties. The fresh yield per unit area showed highly significant differences among the varieties $\left(F=47.68>F_{0.01}\right)$. The difference in sugar content among the 13 varieties reached a significant level $\left(F=4.25>F_{0.01}\right)$, with variety No. 7 from Inner Mongolia having the lowest sugar content and variety No. 6 from Heilongjiang having the highest sugar content. However, variety No. 6 showed no significant difference in sugar content when compared with varieties No. 1, No. 2, No. 5, No. 9, No. 10, No. 11 and No. 13. For the 5 selected important agronomic traits, differences did exist between the varieties.
A cluster analysis was performed for the 13 varieties based on all 31 biological traits using NTSYS-pc software. The results showed that the genetic distance between variety No. 13 (Liaosiza No. 1) and variety No. 10 (BT802) was the smallest (0.787), whereas the distance between variety No. 10 (BT802) and variety No. 7 (Chitian No. 1) was the largest (2.221). A dendrogram was generated based on the genetic distance between the 13 sweet sorghum varieties, which may genuinely reflect genetic differences between varieties (Figure 1). Using an appropriate combined line at GD $=1.558$, the 13 varieties were divided into two groups. The first group is comprised of variety No. 7 (Chitian No. 1) and variety No. 12 (Xingaoliang No. 3), while the other varieties were clustered into the other group. The above results indicate large genetic differences between the two varieties from Inner Mongolia and Xinjiang and all other varieties, while relatively small genetic differences were present between varieties from the same region, such as Liaoning Province.

\subsection{Genetic Polymorphisms and Cluster Analysis Based on RAPD Markers}

PCR amplification was performed using 100 random primers, and 22 primers were shown to have distinct amplification bands and produce stable and reproducible results with obvious polymorphisms. These 22 primers were selected to amplify samples from the 13 sweet sorghum varieties. A total of 144 bands were obtained, and 84 of them showed polymorphisms, with a polymorphism rate of $58.33 \%$. The average number of polymorphic bands per primer was 3.82 .

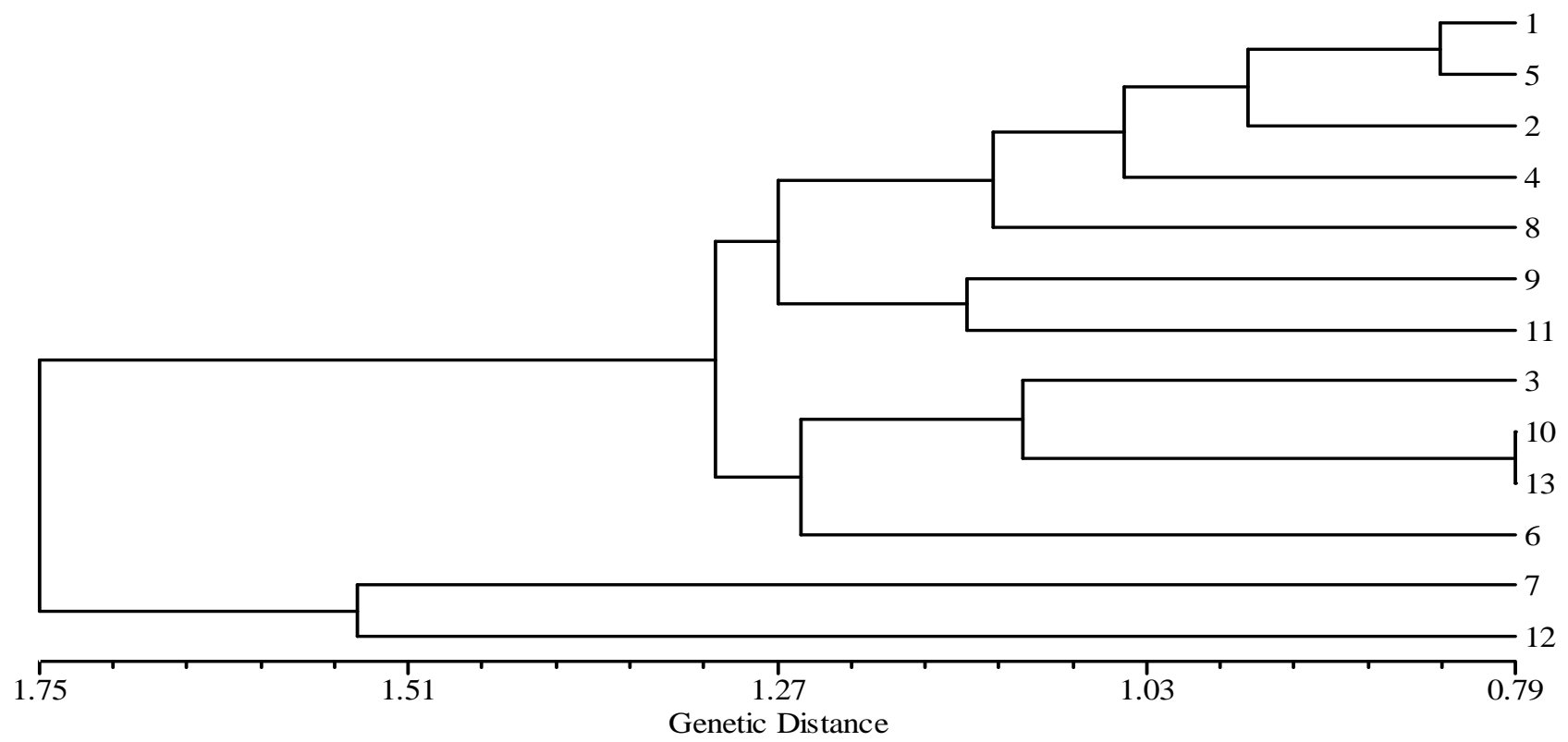

Figure 1. Dendrogram of the 13 sweet sorghum varieties based on 31 biological traits. 
The genetic similarity coefficients among the sweet sorghum varieties were calculated according to the analytical results of electrophoretic band patterns, and a phylogenetic tree was generated based on the calculated coefficients (Figure 2). The 13 sweet sorghum varieties did not show large differences in genetic similarity coefficients, which ranged from 0.694 to 0.896 . Among these varieties, variety No. 2 (Liaotian No. 5) and variety No. 8 (Liaotian No. 7) exhibited the highest similarity, with a similarity coefficient of 0.896 , while variety No. 7 (Chitian No. 1) showed the lowest similarity to variety No. 1 (Liaotian No. 4), with a coefficient of 0.694. As shown in Figure 2, variety No. 7 from Inner Mongolia is clearly separated from the other 12 varieties. If a coefficient of 0.764 is used as the classification standard, all the varieties can be clustered into three groups, with variety No. 7 (Chitian No. 1) in one group, variety No. 6 (Longtian 062) and variety No. 12 (Xingaoliang No. 3) in another group and the other 10 varieties in the final group. The above results indicate that the three varieties from Inner Mongolia, Xinjiang and Heilongjiang exhibited low genetic similarities with all the tested varieties from Liaoning Province, suggesting significant genetic differences among the varieties from different geographic regions.

\subsection{Genetic Polymorphisms and Cluster Analysis Based on SSR Markers}

A total of 79 pairs of primers were selected for this study, including 10 pairs designed in our laboratory from EST or BAC and 69 pairs designed according to the published literature [4,5]. First, 40 pairs of primers that exhibited polymorphisms in the 13 varieties were selected. From among these 40 pairs, 31 pairs (including two pairs of self-designed primers, BAC2 and BAC5) with distinct amplification bands and consistent results were chosen for the analysis of genetic diversity of the 13 tested varieties. A total of 103 bands were amplified from these 31 pairs of SSR primers, and 81 bands showed polymorphisms, with a polymorphism rate of $78.64 \%$.

Genetic similarity coefficients ranging from 0.534 to 0.971 among the 13 sweet sorghum varieties were obtained using the NTSYS-pc software. Variety No. 13 and variety No. 10 exhibited the highest genetic similarity coefficient (GS $=0.971)$, indicating a close genetic relationship between the two varieties. The cluster dendrogram of all the varieties is shown in Figure 3. Using a similarity coefficient of 0.676 as the classification threshold, the 13 sweet sorghum varieties can be clustered into 3 major groups. Variety No. 12 from Xinjiang and variety No. 7 from Inner Mongolia were clustered into one group. However, these two varieties did not show a very high similarity coefficient $(\mathrm{GS}=0.767)$, suggesting the existence of genetic differences between them. Variety No. 6 from Heilongjiang was in one group. The other 10 varieties were clustered into the last group, which includes all the tested varieties from Liaoning Province, which indicates a close genetic relationship between these varieties.

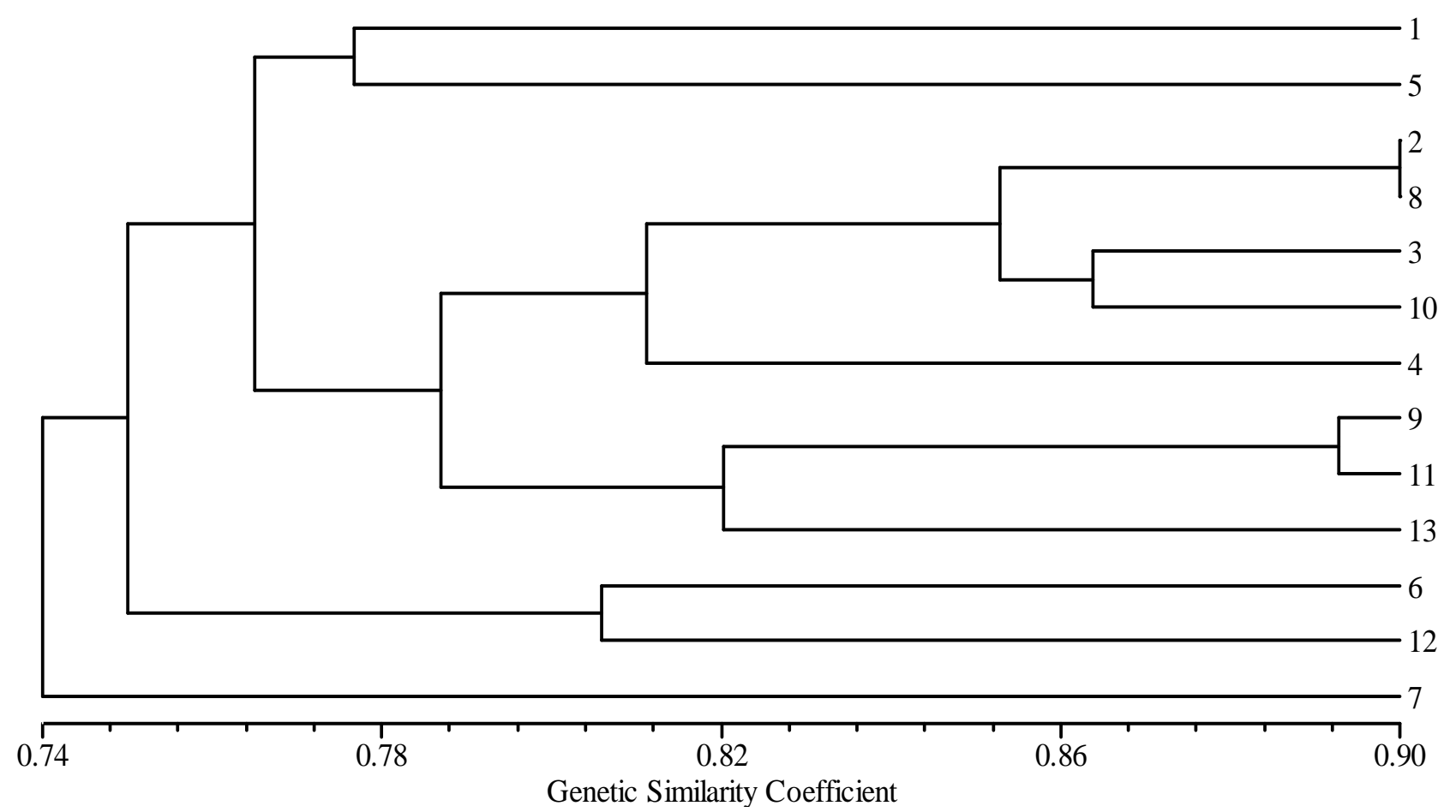

Figure 2. Clustering dendrogram of the 13 sweet sorghum cultivars based on RAPD genetic similarity coefficients. 


\subsection{Cluster Analysis Results Based on Combined Data Analysis}

The distance, DIST, between the 13 sweet sorghum was obtained based on data from the 31 biological traits and 0-1 data from the RAPD and SSR electrophoretic bands The largest genetic distance $(\mathrm{GD}=1.672)$ was observed between variety No. 7 and variety No. 1, while the smallest genetic distance (GD = 0.915) was found between variety No. 2 and variety No. 8, followed by variety No. 10 and variety No. 13 (GD = 0.917). The cluster dendrogram of the 13 varieties was generated according to the distance, DIST (Figure 4). A genetic distance of 1.443 was used as the classification threshold, and all the varieties were clustered into 4 groups. Varieties No. 7, 12 and 6 were clustered alone into three separate groups, while the other 10 varieties were clustered into another group. This result displayed a similar trend with results from the three individual data analyses discussed above for biological traits, RAPD and SSR molecular markers, with some differences. Compared to the analysis of SSR molecular markers, the combined data analysis results classified varieties No. 7 and 12 into two different major

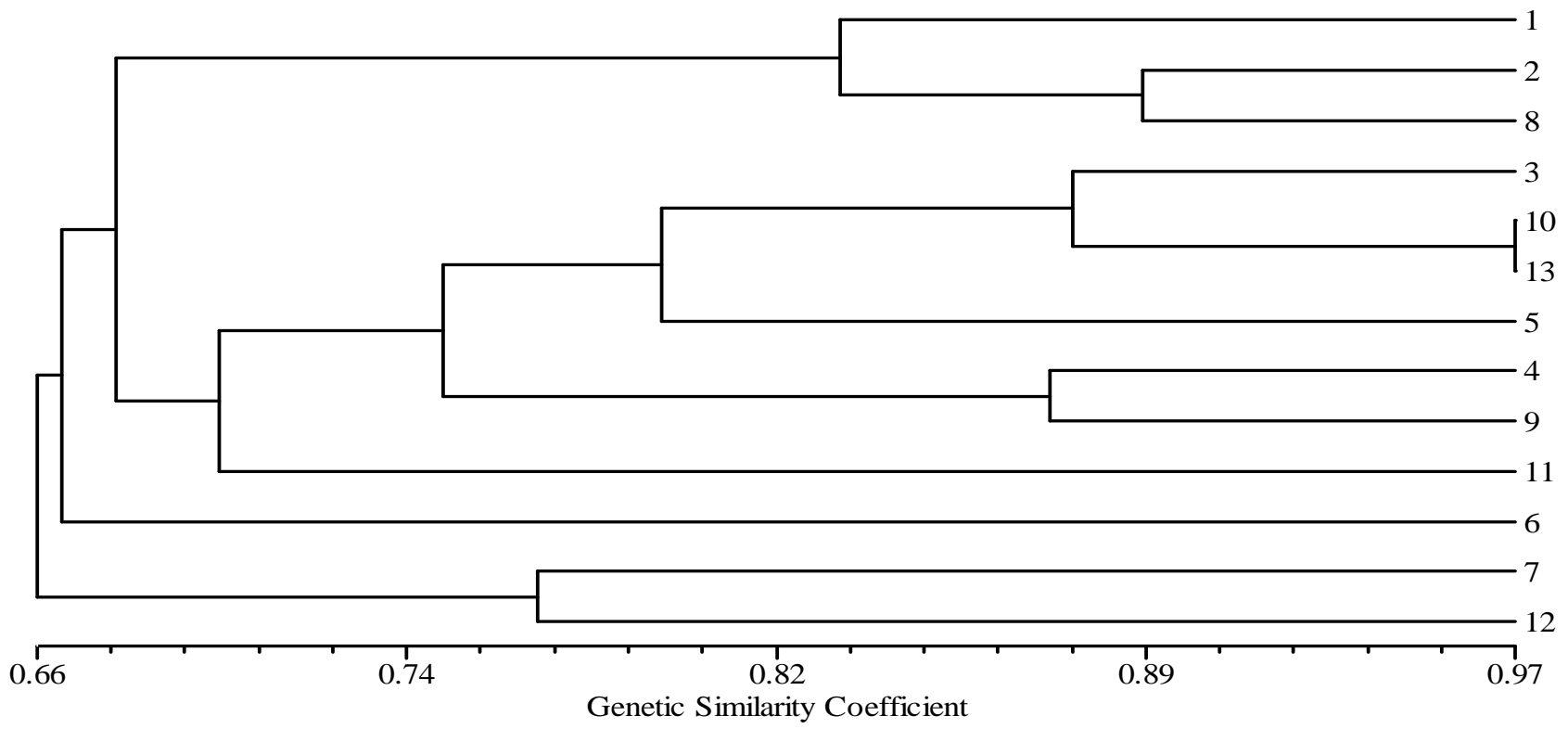

Figure 3. Cluster dendrogram of the sweet sorghum cultivars based on SSR marker analysis.

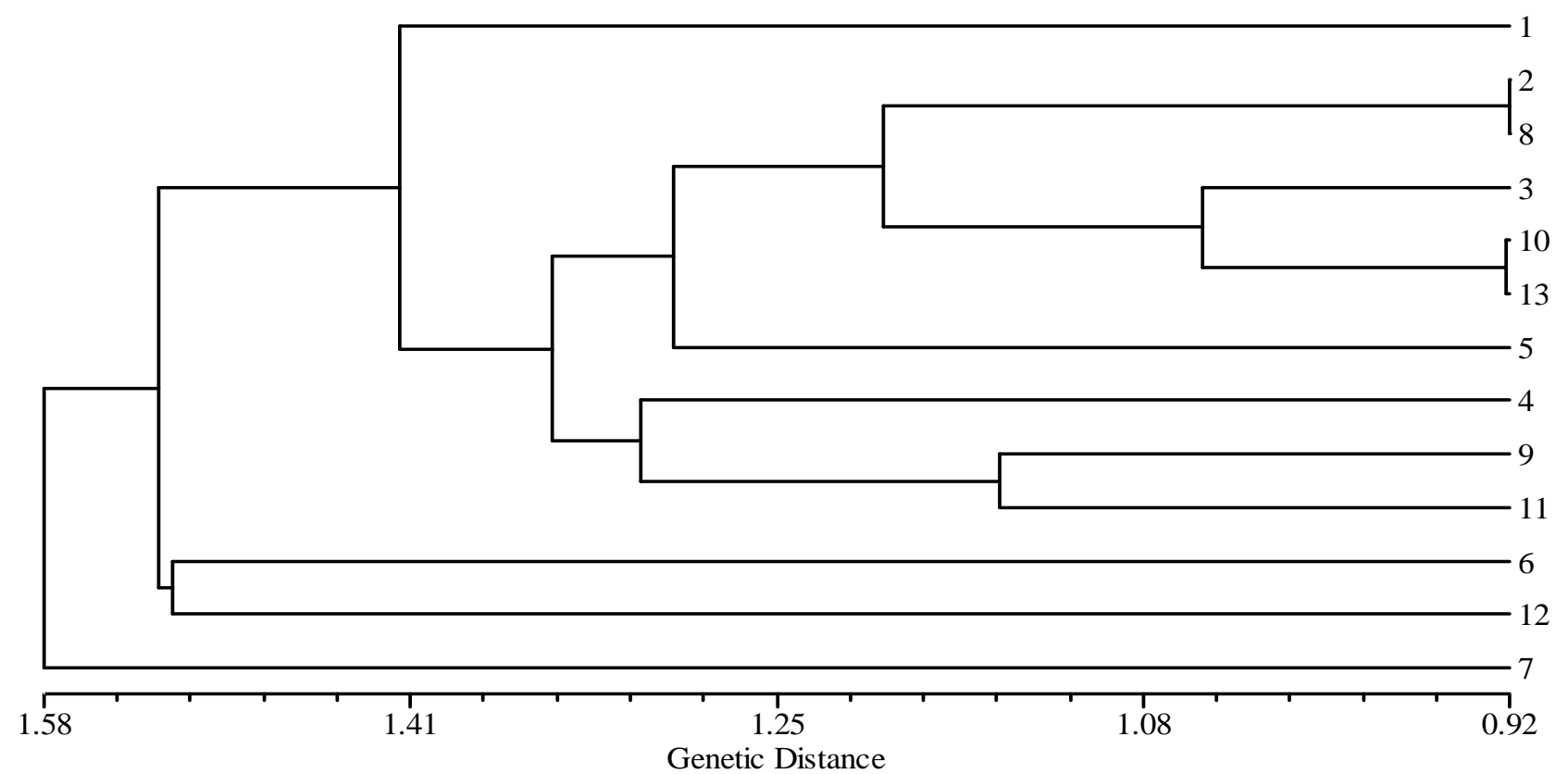

Figure 4. Cluster dendrogram of the 13 sweet sorghum cultivars based on combined data analysis. 
groups $(\mathrm{GD}=1.530)$, indicating that combined data analysis may more objectively reflect the fundamental differences between the varieties. In summary, the varieties currently bred in China exhibited genetic differences because they are from different geographic regions and breeding institutions. However, those varieties from the same region, and especially from the same breeding institution, showed a relatively high level of genetic similarity.

\section{Discussion}

\subsection{Comparison of the Individual Molecular Marker Method and the Combined Data Analysis Method}

Multiple RAPD primers were used to amplify from the same variety for multiple trials in the preliminary experiments of this study. The results were reproducible as long as the source and lot of reagents, the equipment models and the quality of the DNA templates were consistent, and the amount of reagents used and amplification conditions were strictly controlled. This study discovered that RAPD markers can be used to supplement and improve conclusions about the genetic diversity and relationships of sweet sorghum varieties. SSR molecular marker technology has been widely used in studies of crop genetic diversity [4-6]. However, no studies on SSR genetic diversity among sweet sorghum varieties bred in China have been reported. SSR markers were utilized in the present study for diversity analysis of 13 sweet sorghum varieties, and they showed a relatively good correlation with the numerical analysis and RAPD analysis. In the present study, combined data analysis was performed with biological traits, RAPD and SSR amplification band patterns, which may more comprehensively and objecttively reflect the fundamental genetic differences among varieties and better reveal the genetic relationship between sweet sorghum varieties from different regions or from different breeding institutions within the same region.

\subsection{The Genetic Basis for the Sweet Sorghum Varieties Currently Bred in China}

This study showed high genetic similarity coefficients in varieties from the same region, especially those from the same breeding institution. For example, no matter which test method was used, the 5 varieties bred by the Sorghum Institute of Liaoning Academy of Agricultural Sciences were all clustered into the same major group, and varieties No. 2 and 8 were always in the same minor group. Compared with varieties from the same breeding institution, those from different regions or different institutions exhibited larger genetic distances and smaller similarity coefficients. However, surprisingly, some varieties from different breeding institutions showed very high similarity, such as varieties No. 13 and No. 10, with SSR similarity coefficients as high as 0.971 . These two varieties are very likely derived from the same parental variety because the breeding institute for variety No. 10, Shenyang Laoben Seeds Co., was established following its separation from the Sorghum Institute of Liaoning Academy of Agricultural Sciences. There are generally only small genetic differences between the 13 sweet sorghum varieties. This may be because the sweet sorghum varieties currently popular in China are mainly selected and bred at Liaoning Academy of Agricultural Sciences. In addition, the majority of parental varieties at other breeding institutions are from Liaoning Academy of Agricultural Sciences as well. As described above, the breeding of sweet sorghum varieties is a serious situation in China. New sweet sorghum varieties can be created through backcrossing or molecular methods. This would be especially beneficial for the generation of new sterile male varieties of sweet sorghum.

\section{REFERENCES}

[1] Q. W. Zhan, B. F. Wang, X. Xiao, S. B. Wang, Z. P. Li and S. D. Zhang, "Numerical Analysis of Biological Characters in Sorghum [Sorghum bicolor (L.) Moench] and Sudangrass [Sorghum sudanense (Piper) Stapf]," Acta Prataculturae Sinica, Vol. 13, No. 2, 2004, pp. 61-66.

[2] A. P. Rasnitsyn, "Ontology of Evolution and Methodology of Taxonomy,” Paleontological Journal, Vol. 40, No. Supplement 6, 2006, pp. 679-737.

[3] J. G. K. Williams, A. R. Kubelik and K. J. Livak, "DNA Polymorphisms Amplified by Arbitrary Primers Are Useful as Genetic Markers,” Nucleic Acids Research, Vol. 18, No. 22, 1990, pp. 6531-6535. doi:10.1093/nar/18.22.6531

[4] L. Kong, J. Dong and G. E. Hart, “Characteristics, Linkage Map Positions, and Allelic Differentiation of Sorghum bicolor (L.) Moench DNA Simple-Sequence Repeats (SSRs)," Theoretical and Applied Genetics, 2000, Vol. 101, No. 3, pp. 438-448. doi:10.1007/s001220051501

[5] S. J. Schloss, S. E. Mitchell, G. M. White, R. Kukatla, J. E. Bowers, A. H. Paterson and S. Kresovich, "Characterization of RFLP Probe Sequences for Gene Discovery and SSR Development in Sorghum bicolor (L.) Moench," Theoretical and Applied Genetics, 2002, Vol. 105, No. 6, pp. 912-920.

[6] Q. W. Zhan, T. Z. Zhang, B. H. Wang and J. Q. Li, "Diversity Comparison and Phylogenetic Relationships of $S$. bicolor and S. sudanense as Revealed by SSR Markers," Plant Science, Vol. 174, No. 1, 2008, pp. 9-16. doi:10.1016/j.plantsci.2007.09.007

[7] Q. W. Zhan, J. Q. Li, B. H. Wang and Y. F. Li, "Establishment of DNA Fingerprinting for 42 Sorghum and Sudangrass Accessions and 2 Sorghum-Sudangrass Hybrids," Acta Prataculturae Sinica, 2008, Vol. 17, No. 6, pp. 85-92.

[8] F. J. Rohlf, "NTSYS-pc, Numerical Taxonomy and Multivariate Analysis System,” Applied Biostatistics Inc., New York, 2000. 